Transactions of the Royal Society of Edinburgh

http://journals.cambridge.org/TRE

Additional services for Transactions of the Royal Society of Edinburgh:

Email alerts: $\underline{\text { Click here }}$

Subscriptions: $\underline{\text { Click here }}$

Commercial reprints: $\underline{\text { Click here }}$

Terms of use : $\underline{\text { Click here }}$

\title{
VII.-Notes on the Dynamics of Cyclones and Anticyclones
}

John Aitken

Transactions of the Royal Society of Edinburgh / Volume 40 / Issue 01 / January 1905, pp 131 - 156

DOI: $10.1017 /$ S0080456800034244, Published online: 06 July 2012

Link to this article: http://journals.cambridge.org/abstract S0080456800034244

How to cite this article:

John Aitken (1905). VII._Notes on the Dynamics of Cyclones and Anticyclones. Transactions of the Royal Society of Edinburgh, 40, pp 131-156 doi:10.1017/S0080456800034244

Request Permissions : $\underline{\text { Click here }}$ 
VII.-Notes om the Dymemies of Cyclome's and Anticyclones. By John AITken, F.R.S. (With a PJate.)

PART I.

(Rinl March 5, 1900.)

The vertical movements of the earth's atmosphere from which the energy is derived which causes the horizontal movements of the air which we call winds, and by means of which the moisture evaporated from the surface of land and water is collected and carried to the higher regions of the atmosphere, where it is condensed to cloud and again distributed in the form of rain over the earth's surface, are of great interest, and a thorough knowledge of the laws governing these vertical movements is necessary to enable us to arrive at a correct forecast of the coming weather over any area.

In the present communication I do not intend entering on a review of the work which has already been done in this field. Mauy explanations have been offered of the movements of cyclones and anticyclones as a whole, aud of the winds within their areas, but any detailed reference to these would far exceed the limits of these notes, and would, I fear, only complicate matters. In what I have to say there will necessarily be much that is old, and I am sorry I must leave to the reader the task of finding out what is new, as in a subject of this kind, on which so much has already been written, it is impossible to say whether any particular point has not been referred to before by some other writer. And further, I shall confine my remarks to what takes place over our area and Vestern Europe, so as to avoid unnecessary verbal complications; but the principles can be easily applied to other areas in the Northern hemisphere, and to the reversed direction of circulation in the cyclones and anticyclones in the Southern hemisphere.

At the outset it will be as well for me to make a few elementary remarks on the formation of cyclonic movements, as I find that many who take an interest in Meteorology have rather hazy ideas of how the vortex motion in cyclones is produced. All that some seem to think necessary is to have an area of low pressure and that the air will rush towards it in spiral paths, just as they see water in a wash-hand basin forming a vortex movement whenever the plug is withdrawn and the water allowed to run away. Now it must be clearly understood that no vortex will form in iir or water that is at rest before the low-pressure area is formed; the air or water under these conditions will flow to the low-pressure centre along radial paths and not in spiral ones. The above statement requires qualification. When it is said the air or water is at rest it is not meant that it is at rest absolutely, which would be an impossibility in this rotating, revolving, and space-travelling world of ours. All that is necessary is that the water or VOL. XL. PART I. (NO. T). 
air be at rest relatively to the centre of low pressure-that is, that the centre of low pressure and the air surrounding it are all moving in the same direction and at the same velocity. Another qualification necessary in the case of the air on the earth's surface is, that the area of low pressure be very small, otherwise the different parts of the area will have different rates of movement owing to the earth's rotation causing its surface and the air near it to move faster near the equator than near the poles, so that the air to the south of the low-pressure centre moves more quickly in an easterly direction than the air to the north of it-that is, supposing there is no wind.

Let us now return to the question of the cause of the cyclonic movement. That motion relatively to the low-pressure centre is necessary to produce the spiral approach of the air or water to the centre is easily illustrated. Take a circular vessel-say $40 \mathrm{~cm}$. in diameter and $15 \mathrm{~cm}$. deep-filled with water. The vessel should have an opening in the centre which can be closed from the outside, so that it can be opened without disturbing the water in the vessel. Put some sawdust, or similar substance, in the water and mix it with it. Now leave it at rest till all movement in the water ceases, then open the outlet in the bottom of the vessel and allow the water to run away, when it will be seen that the sawdust suspended in the water moves towards the outlet flowing from all directions, and at all depths, in radial lines, without a trace of vortex movement.

Let the conditions be now changed and a slight circular motion be given to the water before the outlet is opened. A well-formed cyclone will now be formed by the outflowing water, and the quicker the initial movement the greater will be the number of turnings in the spiral path of the water before it arrives at the outlet, and the deeper the bollow in the surface of the water over the outlet, while the direction of the spiral movement will be the same as the initial movement given to the water. The depression of the surface of the water at the centre of the vortex is extremely interesting. If the water before the outlet be opened be not perfectly at rest, a faint depression will always be detected over the outlet, having in its centre a quickly-rotating vortex of very small area. If, however, the initial motion be greater-but it need not be quick-then the depression deepens and forms an air-tube extending to the bottom of the vessel, showing that the slow initial circular motion has enabled the low-pressure area to generate a velocity at the centre of the cyclone sufficient to enable the centrifugal force of the water at that part to withstand the hydrostatic pressure at the bottom of the vessel.

On examining these water vortexes there is a point that strikes one as very remarkable-namely, the great increase in the velocity of the water as it approaches the centre of movement. Not only is the angular velocity greatly increased owing to the shorter path required to complete a revolution near the centre, but the absolute velocity is also greatly increased. Perhaps this point can be more easily seen by making the experiment in another form, and using solids in place of liquids. Suspend two balls, A A, fig. 1, by long fine wires, either from the same point of suspension, or, to reduce the 
complications due to the effects of gravity owing to the balls falling a short distance as they approach the centre, suspend the balls from the opposite ends of a short beam B, suspended by fine wires, C ( ) as shown in the figure. The upper ends of the wires are fixed to a swivel $D$, to enable the balls to be put into circular movement round their common centre. To each of the balls a short cord $E \mathrm{E}$ is attached. These cords are passed through a small ring $F$, which is suspended at the level of the balls by the wire $G$ fixed to the swivel 1). The two cords $\mathrm{EE}$ are joined below to another swivel $H$, which has a cord attached to its lower end, to allow the balls to rotate round $G$, whilst the lower cord is held in the hand. If we now put the balls into a slow circular movement round their common centre, and take hold of the lower central cord below the swivel $\mathrm{H}$, note the rate of revolution and pull the cord downwards. This will draw the balls towards the centre of motion, in the same way as the particles of water are drawn into the centre of the vortex. When this is done it will be seen that the initial slow motion is rapidly quickened, and that when the balls are near the centre they are whirling round each other at a great velocity.

Another way of making this experiment is to remove the small ring $\mathrm{F}$ and its suspending wire, and tie the balls with a cord of about $50 \mathrm{~cm}$. long, then attach to this cord, at a point halfway between the balls, a small rod held vertically. If the balls be now made to rotate round the rod, and the rod be prevented from turning, the balls will gradually wind themselves towards the centre, and it will be noticed when making the experiment in this way that whilst the angular velocity increases, the absolute velocity does not, showing that the mere fact of the balls

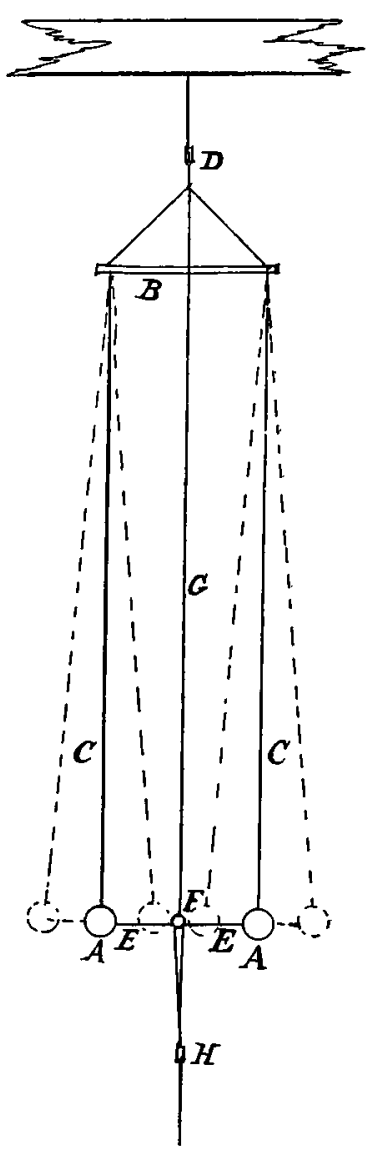

FIG. 1. approaching the centre has nothing to do with the increase in the velocity observed in the previous experiment. If now, in place of holding the centre rod firm, we cause it to rotate on its vertical axis so as to wind the balls towards the centre, it will now be seen that the absolute as well as the angular velocity is greatly increased, as was seen in the previous experiment, in which the balls were drawn together by means of the cords $\mathrm{E} \mathrm{E}$. The balls now fly round so quickly the eye can hardly follow them.

Further, it will be noticed that the balls offer a rapidly-increasing resistance to the centripetal force. This to a certain extent is to be expected, because as the balls approach the centre the centrifugal force increases; if the velocity is constant it is double what it was at first by the time the balls are drawn half way to the centre. But on account of the increased velocity which we cannot avoid giving the balls in the process, they are enabled to offer still greater resistance to the centripetal force. This, 
which might be called a secondary result, comes in and assists the centrifugal force in offering resistance to the change. There are plenty of similar results in physical phenomena, where these secondary results assist in resisting the change, as for instance, when we compress air. Suppose, as in the above case, we reduce the volume to one-half, then we have doubled the pressure, which seems fair enough. But the air, with almost human-like dislike to compulsion, heats in the process, and by so doing helps to resist the compression till it has had time to cool. These remarks may suggest something as to the great power of vortex movements for storing energy.

The cause of the great increase in the velocity in the balls when they are drawn towards the centre is due to work having been done on them. When the balls are revolving round their common centre, held by the cord at a fixed distance, no energy is communicated to them by the tightened cord, because the force acts at right angles to the direction of movement. But when the balls move in a spiral path, then only one component of the radial force acts at right angles to the direction of motion, whilst another is in the direction of the movement, thus tending to increase its velocity. The same explanation applies to the increased velocity of air or water approaching the centre of cyclones.

This resistance offered by the spirally-moving air to be drawn into the centre of the cyclone has one important effect. It enables the cyclone to develop more energy than if the air moved inwards radially. When the air moves in radially the supply is so abundant that a great fall of pressure cannot take place, but when the inflow of the air is retarded by the centrifugal force, a greater fall of pressure results, and the energy of the cyclone is increased; and in addition to this we shall see later that it adds greatly to the efficiency of cyclones considered as circulating engines.

It may perhaps be as well that I give my reasons for saying that whilst the vortex motion gives rise to strong currents-that is, increase of velocity-it yet retards the inflow, and by so doing it will allow a lower pressure to be formed in the centre. This point can be most easily illustrated by means of water vortexes. The circular vessel already referred to was used, the outlet being a short pipe fixed in the centre of the vessel. In each experiment the vessel was filled with water to the same depth, and the time observed that the water took to empty when it had no motion before starting to empty-that is, emptied out without vortex motion-and when a slight movement was given so as to cause it to form a cyclone when emptying. The result was the water took a half longer in the latter case than in the former, showing a great retardation due to the cyclonic motion, and this retardation results in a lowering of the pressure at the centre, and it also prevents the low-pressure area being so rapidly filled up. Care was of course taken in the above experiments that the pipe carrying away the water was always full in both cases, so that the head of water should be the same in both. This was done by placing a glass disc over the outlet and at some distance above it, and making the outlet pipe discharge 
below water. By these means air was puvented entering either end of the discharge pipe.

For making experiment on the cyclonic movenents in air, I have found the following piece of apparatus useful. To produce the up-draught a thin metal tube $15 \mathrm{~cm}$. diameter and fully $2 \mathrm{~m}$. high was used. At the lower end of the tube is fixed a circular dise $76 \mathrm{~cm}$. diameter. The dise is supported on three legs, $15 \mathrm{~cm}$. high, thus leaving an air space of $15 \mathrm{~cm}$. between the disc and the table on which it resti. To produce the up-draught three small jets of gas are fitted inside the tube near the lower end. 'To study the circulation produced by the apparatus, a number of small light vanes supported on small stands were used, so that they could be put to show the direction of the air currents anywhere within the area affected. These vanes show the directions of the circulation at the different parts of the area affected by the cyclone, but for studying the variations in the circulation at different heights from the surface of the table, the fumes from hydrochloric acid and ammonia were found to be more useful. These fumes are very suitable for the purpose, as they have only a very slight proper motion of their own, rising only very slowly, so that their own movements do not interfere much with the cyclonic motions. When working with fumes a large plate of glass should be placed below the apparatus to give freedom for experimenting, and the fumes are best made by placing pieces of lixper on the glass and dropping the ammonia and acid on them. These papers, being close to the surface, do not give rise to local eddies.

Before lighting the gas to make an experiment, some fumes should be made under the tube, to see if there are any air currents in the ronm. Suppose there are none, and the fumes rise slowly without drifting in any direction, the gas should now be lighted and a number of centres of fumes should be started at different points under and round the tube, either by dropping the acid and ammonia on separate pieces of paper, or by putting them in watch glasses. If we olsserve the fumes under these conditions, it will be seen that they rise and move radially towards the hot chimney, moving upwards in even curves, but showing no tendency to rotate round the centre. An element is still wanting to produce the cyclonic motion, the air must be given some initial movement before the up-draught will take the spiral form. Suppose that on first testing the air on the table, we found that it was not free from movement, but that there was a slight current blowing across it. If this current be equally strong at all points, it will not be of much use in generating cyclonic movement, but if we put up a screen so as to cut off the current from one side of the area and allow it to blow on the other, then we have the conditions necessary for producing vortex motion under the chimney. If we examine the fumes rising under these new conditions, it will be seen they no longer move radially but are in violent cyclonic motion, swirling round and round in the direction given by the tangential current, the rising fumes forming graceful ascending spirals. So strong is the circular motion that at times the gas in the chimney is heard flaring as in i strong wind. 
If there is no cyclonic motion formed when the gas is lit, any light objects lying on the table are not disturbed by the radially moving air, but if a good cyclonic circulation is set up, then any light bodies, such as sawdust, paper, or tufts of cotton wool, which happen to be lying under the chimney, are seen to be lifted up and tossed about, generally getting thrown out of the centre of the cyclone by their centrifugal force, but the cotton wool is frequently drawn up the centre and discharged at the top of the tube. Should there be no current in the room suitable for producing the tangential motion, then the cyclone may be formed by blowing by artificial means. across one side of the area, or over two or more sides if in correct directions.

Let us now study more closely the movements of the air under the draught tube. Suppose we have it arranged so that a gentle current is blowing across one half of the cyclonic area, and the air is forming a cyclone under

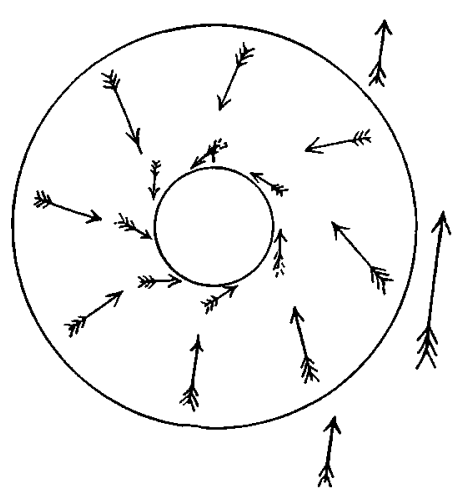

FIG. 2. the tube, we shall now place the small vanes-already referred to-within the area of the cyclone, to show the directions of the movements of the air-that is, the winds at the different parts of the cyclone. The result is roughly shown in fig. 2. The air current over the table is supposed to be blowing in the direction of the arrow at the side, and to be strongest on that side of the area, and the cyclonic movement is roughly indicated by the arrows in the diagram. It is impossible to get these directions satisfactorily indicated by the vanes, owing to the difficulty of keeping the cross current constant, and the amount of curving of the spirally-moving air is constantly changing with every change in this tangential component. The arrows, however, show that on the side where the tangential current is strongest the in-draught is most tangential, whilst on the opposite side the incoming air moves more radially, or, to put it more generally, the air in the right-hand front of cyclones in our area moves more tangentially than the opposite left-hand rear position. It should be noticed here that these remarks apply to the case where the centre of the cyclone is fixed. Some modification will be necessary when applying them to cyclones in which the centre of low pressure is in motion.

We may learn something further with this apparatus if we use fumes to study the movements of the air over the different sections of the cyclonic area. One very marked result which will be observed is that the air-as shown by the fumes-near the surface of the table at all parts of the area moves much more radially than the air higher up, and also that the air lying on the surface of the table is drawn into the very core of the cyclone, up which it rises in a rapidly-circling path of small diameter, whilst the air higher up comes towards the centre along a rising wider spiral path, and forms the outer lining of the cyclonic tube. The lower air keeps near the surface till it arrives near the centre of the cyclone and then rises, making many more revolutions than the 
outer air for the sime amount of ascent, as roughly indicated in fig. 3. This indraught of the lower air into the core of the cyclone is one of great importance in connection with meteorological phenomena, and will be referred to later on. The reason for the lower air being hriwn into the core is very obvious. The air near the cromul, or surface of the table in this case, has less tangential movement thin the air ligher up, owing to its motion being retarded by friction. The result of this is that whilst the greater centrifugal force of the upper air keeps it back against the low pressure in the crclone, the lower air as it moves nearly radially offers but

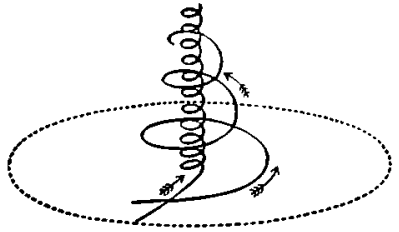

FIG. 3. little resistance to the in-draught. Hence the lowest stratum of air being at the lower end of the cyclonic tube, it is drawn into the very centre.

Anyone can make similar experiments to these without a special draught tube, and study for themselves the conditions necessary for the formation of cyclonic circulation. All that is necessiry is a good fire, with a free-going chimney, and a wet cloth. The cloth is hung up in front of the fire and pretty near it, so as to cause a liberal amount of condensed steam to rise from its surface. Probably without some arrangement of the draughts in the room no cyclone will be formed, and the vapour will rise vertically, keeping close to the wet cloth. But if the room has a door or a window in the wall at right angles to the fireplace, and at the side nearest the fireplace, so as to cause the air coming from it to make a cross current past the fire, then a cyclone will be formed and the steam on the wet cloth will be seen circling round, and when the cyclone is well formed, all the steam is collected into the centre of the cyclone and forms a white pillar extending from the cloth to the chimney. If the cross-draught be equally strong at top and bottom of the wet cloth, it will be necessary to screen the current from either the top or bottom of the cyclonic area. Should there be no suitable draught in the room, then an artificial one may be made in any direction across one side of the wet area.

We have seen from these experiments that no cyclone can form without some tangential movement in the air entering the area of low pressure. The next question one vaturally asks is-Has this tangential motion any other effect on the cyclone? From the fact that some of the air entering the cyclone has motion in a particular direction, we would naturally expect that unless an equal amount of air, moving in the opposite direction, also entered at the same time on the other side, that the whole system would move in the direction of the greatest tangential force. If we examine the cyclone produced in our artificial conditions with the draught tube we shall find that this is the case. The lower end of the cyclone bends away from under the centre of the apparatus, moving in the direction of the tangential current.

This point can, however, be better illustrated by means of water vortexes. Using the circular vessel as before, and in order to make the tangential current on one side stronger than on the other, all that is necessary is either to put the outlet for the 
water excentric to the walls of the vessel, or more easily by taking a strip of thin metal, having a breadth the same as the depth of the vessel and of a length of a little more than the diameter, and bend it into its place in the circular vessel, as shown in fig. 4. When the water now runs out it all passes round the

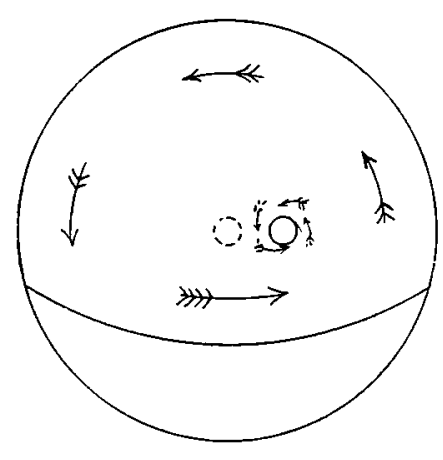

Fig, 4. vessel, and as the passage between the outlet, that is the centre of the cyclone, and the temporary division is narrower than at any other place, the water has to pass this part at a much greater velocity than at any other place, and the result is, the top of the vortex no longer remains over the outlet, but travels in the direction of the quickest moving water, showing that, as might be expected, the quickest moving water tends to carry the centre of the vortex along with it. See fig. 4, where the small dotted circle represents the outlet and the full circle the top of the vortex, which is carried by the water to the right, the direction of movement of the water being shown by the arrows. In addition to moving in the direction of the strongest current, the centre seems also to be attracted towards the quickly-moving area, but to this point I shall not further refer at present.

Turning now from these experimental obserrations, let us see how far they help us to understand the phenomena in cylones and anticyclones in our atmosphere. At the outset I may say that in forecasting attention seems to have been given too exclusively to what takes place in cyclonic areas, and too little to the part played by anticyclones. We have been looking too much on the cyclone as the active member of this dual partnership. But I think we will have to admit that the anticyclone is not the sleeping partner it is generally supposed to be, whose only duty is to follow and fill up the depressions made by the active partner. A closer examination of the part played by the anticyclone will show that it also is an active though silent partner in the firm, and that it initiates and keeps up its own circulation, and collects and forwards the material with which the more showy partner, the cyclone, makes its display. The anticyclone also in a great measure directs the path of the cyclone and adds much to its cyclonic motion. In fact the vertical circulation seems to be liept up by the partners in this dual system playing into each other's hands, and neither could work efficiently without the other.

Let us now look at what the effect would be if there were no cyclones or anticyclones in our atmosphere. Suppose a large area, say, some hundreds of miles in diameter, on the earth's surface over which the air was still, suppose further that the sky was cloudless and a summer's sun was shining. After a time the conditions would become unstable and columns of air would begin to ascend at many points all over the area wherever the air happened to be most highly heated and charged with vapour. The result of this would be a disorderly mob of ascending currents without leader, organization, or combined effort. These small isolated attempts at vertical 
movements could never give rise to anything like a systematized and law-abiding circulation, though they might result in local disturbances such as thunderstorms and rain.

Let us now suppese the conditions are changed and that an anticyclone is blowing at one side of our suppusel hot area, and note the change. When the upper air descends to the carth's surface, it sprearls out in all directions, and if the earth had no motion of rotation the air would spread in radial paths from the centre-that is, supposing the descending column of air had no motion of its own, which is very unlikely. But as little is known on this subject, we must at present neglect it, and may safely do so, as probably even if it has motion it will not greatly affect the result. Suppose that the descending air arrives at the surface of the earth and spreads outwards in all directions, then, owing to the earth's rotation, the air in place of moving radially from the centre, goes in spiral paths. This is owing to the air that moves in a northerly direction blowing over a part of the earth that has a slower easterly motion than the descending air which has come from the south, and the wind seems over the northern part of the anticyclone to blow more or less from the riest. On the other hand, the air moving southwards of the centre flows over an area going more quickly eastwards than the descending air, and the wind over that part of the anticyclone seems to blow more or less from an easterly direction, whilst the air moving east and west from the centre would appear only to have its velocity altered, were it not for other causes which come into play, and make the circulation from the anticyclone to be, in a general way, spiral all round the centre. As the air to the north of the centre moves eastward, and that to the south westwards, the circulation in the anticyclone is right-handed-that is, in the direction of the hands of a watch with its face upwards.

One effect of spiral circulation, to which we have already referred in the experimental part of the paper, is that the air near the surface of the ground where its motion is retarded by friction moves more radially than the upper air. This causes the higher air currents to cross the lower at a greater or less angle, and has the effect of checking the rising of the lower air even when lighter than the upper. In our atmosphere the case is more complicated than in the experiments, owing to the earth's rotation. Over certain areas of the anticyclone the upper and lower airs may be moving in nearly the same direction: this will be the case when the upper air is moving in nearly the same direction as the surface of the earth at the place. But even when the direction of the upper and lower airs is the same, the difference in velocity of the two has some influence in checking the lower air from rising.

Let us now see what the effect of such an organized circulation is in our atmosphere. Let the sketch fig. 5 represent an imaginary anticyclone, which we have shown circular, though the shape is generally far from being so regular. If such a system be established over our imaginary area of sun-heated air, then it will be seen that the anticyclonic winds will prevent the formation of local cyclones, and

VOL. XL. PART I. (NO. 7). 
drive all the moist hot air to its circumference, keeping it near the ground. Suppose, now, that the hot air begins to form a cyclone at the outside of the anticyclone, as shown in fig. 6 , it will be evident it has a supply of hot air collected for it by the anticyclone. But not only has the anticyclone collected the material for making the cyclone, but it also supplies the cyclone with the tangential force necessary for producing the spiral circulation so well known in cyclones.

The explanation generally given of the spiral movement in cyclones is, that the air drawn in from the south has a greater amount of eastward motion than the centre of the cyclone, whilst the air from the north has less, and that these oppositely moving airs from the two directions cause the whole system to circulate round the centre. This, no doubt, is one cause of the circular movement in cyclones, but it is far from

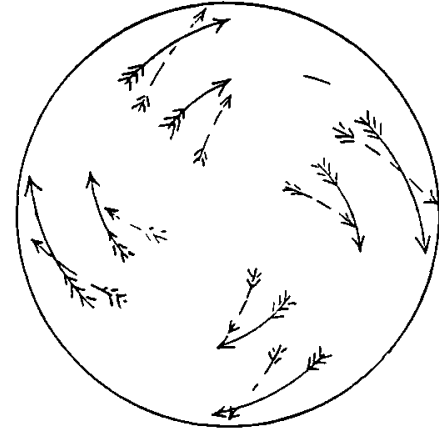

FIG. 5.

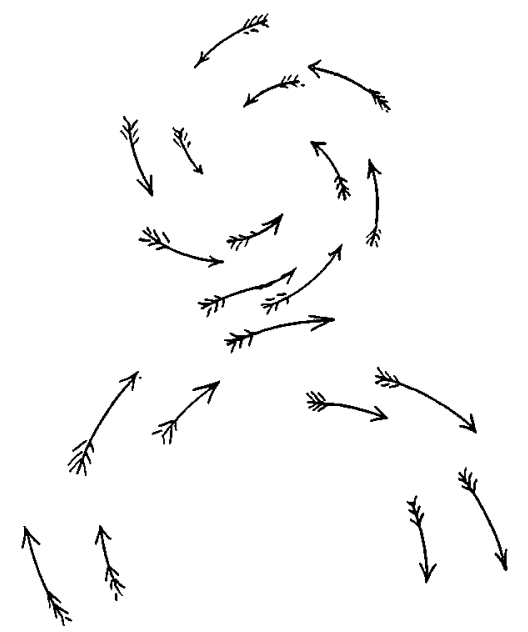

FIG, 6.

being the only one. This tangential action--due to the earth's rotation-only comes into play after the air has travelled a considerable distance towards the centre, whereas the air from the anticyclone already has a considerable tangential movement over the area between the cyclone and the anticyclone. The true explanation would rather appear to be that the cyclone and the anticyclone form one system; the anticyclone forces its air tangentially into the cyclone, whilst the cyclone draws air tangentially from the anticyclone. The earth's rotation is the original source of the rotatory movements, and starts the machine, but both intensify the initial motion; they are, so to speak, geared into each other and kept in motion by the earth's rotation, but both capable, when conditions are favourable, of developing energy on their own account and increasing their rate of rotation.

We saw in the experimental part that a cyclone in the conditions represented in fig. 6-that is, with strong winds blowing tangentially on one side-camnot remain in the same place, but must move in the direction of the strongest winds. It would be fatal for a cyclone to remain long in one place; it would soon use up all the supplies, and when all the hot moist air had been drawn in, it would be starved and get weaker, 
and the depression soon gat filled up. So the cyclone bas to move on for fresh supplies, and if our experimental illustration is corect, it will move along in the direction of the strongest tangential winls.

Now, if we look at the Synopti: Charts issued by the Meterological Office, we shall see that they support this cxplanation, that these tangential winds are the principal canse of the movement of the cyelone as a whole, that the centre of depression moves in the direction and nearly parallel to these winds; or, in other words, the crolone mover nearly pirallel with the isolnars at the side on which the barometric gramlient is strepest. Further observation will show that the greater the difference in the barometric gradients on the two sides, the quicker is the advance of the cyclone, due to the tangential energy on the steep side being greater than on the other. The examination of these charts will also show that, whenever the isobars surrounding the crelone become equally spaced on all sides, that is, when the barometric gradient is the same all round the centre-or even on the front and sides-that the cyclone travels very slowly and is generally in a dying condition. The air under these conditions comes to the cyclone with equal velocities from all dircetions, and there is no tendency for the centre to move. The tangential force is still in great abundince, the winds blowing in from all directions systematically all round, but there is no unbalanced tangential force, and the centre remains over the sime area, and as the hot moist air-the source of the energy -is som used up, the winds tend to fall and the depression to fill up. This sems to be the history of many of the cyclones with circular and concentrically placed isolarm, though in winter they seem occasionally to linger for a time, their winds then seem to depend a good deal on the energy of surrounding anticyclones. It also seems possible that the movements of such cyclones may be determined a good deal by differences of temperature over the anticyclonic areas.

In illustration of these points we shall now refer to some actual examples taken from the Synoptic Charts issued weekly by the Meteorological Office, London. These charts show the distribution of pressure and direction of winds over Europe from observations taken at 8 a.m. and 6 p.m., the day interval being thus 10 hours, whilst the night one is 14 hours. There are charts also giving the temperatures at 8 a.m. Plate I. is a reproduction of four of these charts, showing the distribution of pressure over our area during the passage of two cyclones, - the one a typical quickly-moving one, and the other a slowly-progressing one. In the charts for 10th December 1898 we have the characteristic distribution of pressure, as shown by the isobars, for a quickly-moving cyclone; whilst the charts for 9th December 1897 show the form of isobars associated with slowly-moving ones. In the 1898 cyclone it will be observed that the barometric gradient to the south of the centre of the cyclone was very steep in the 8 a.m. chart, as shown by the closeness of the isobars on that side, whilst the gradients on the other side and in front were easy. The strong winds, as the form of the isobars would lead us to expect, are all to the south of the centre, and are blowing from the west, and the result was that the centre of this cyclone, 
which was over the Shetland Isles at 8 a.m., travelled to the east coast of Sweden by 6 p.m., a distance of 700 miles. During the following night the centre moved in an E.N.E. direction at a considerable velocity, but at a slower rate than during the day, as the barometric gradients at 6 p.m. would lead us to expect.

Turn now to the chart for the 9th December 1897. Here it will be seen that the barometric gradients are nearly equal all round, and strong winds are blowing in all directions systematically round the centre. Now note the slow rate of progress. This cyclone appeared on the N.W. of Scotland on the evening of the 8th December. Next morning at 8 o'clock it was in the place shown in the chart, having travelled a distance of 175 miles in the 14 bours. When this cyclone first appeared the barometric gradient was steeper to the south than on any other side. This, no doubt, was the cause of the advance of 170 miles during the night. It will, however, be seen from the 6 p.m. chart, that the centre moved very little during the day, somewhere about 75 miles, and the whole of the following night it was still over the same place, having apparently become stationary, as it was still there on the morning of the 10th, but the gradients were getting easier, the depression getting filled up. These two cyclones were selected-out of many having similar histories-to illustrate the point under discussion, and they were selected because they travelled over exactly the same track, and at the same time of the year, so that we should only have the question of the conditions shown by the shape of isobars to consider.

To illustrate this point still further, the following table gives in brief the histories of a few of the cyclones during the last two years. The table shows the date and hour when the cyclone first appeared on the chart. Then there is given the situation of the centre of the cyclone, followed by the character of the isobars or distribution of gradient, and, lastly, the distance travelled in miles.

1898.

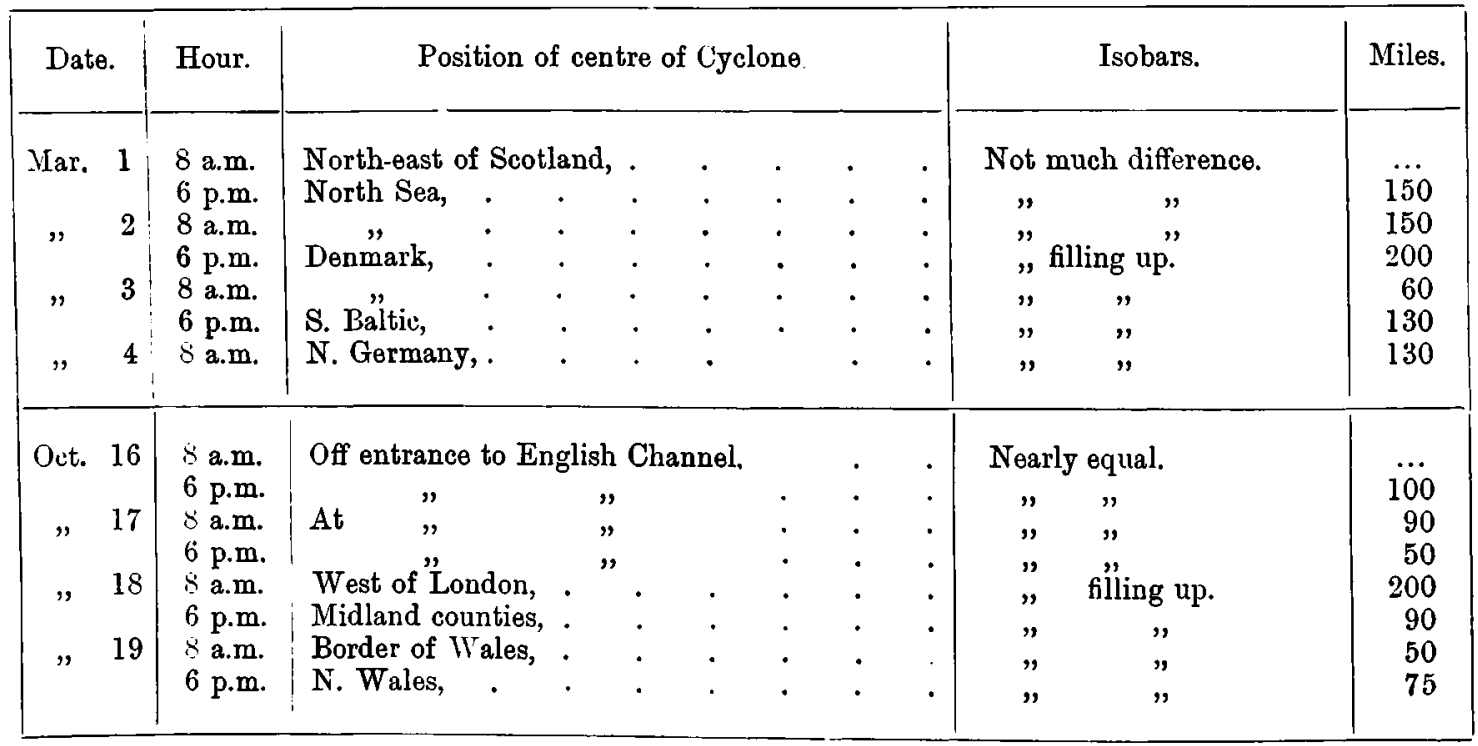


$1 \times 98$.

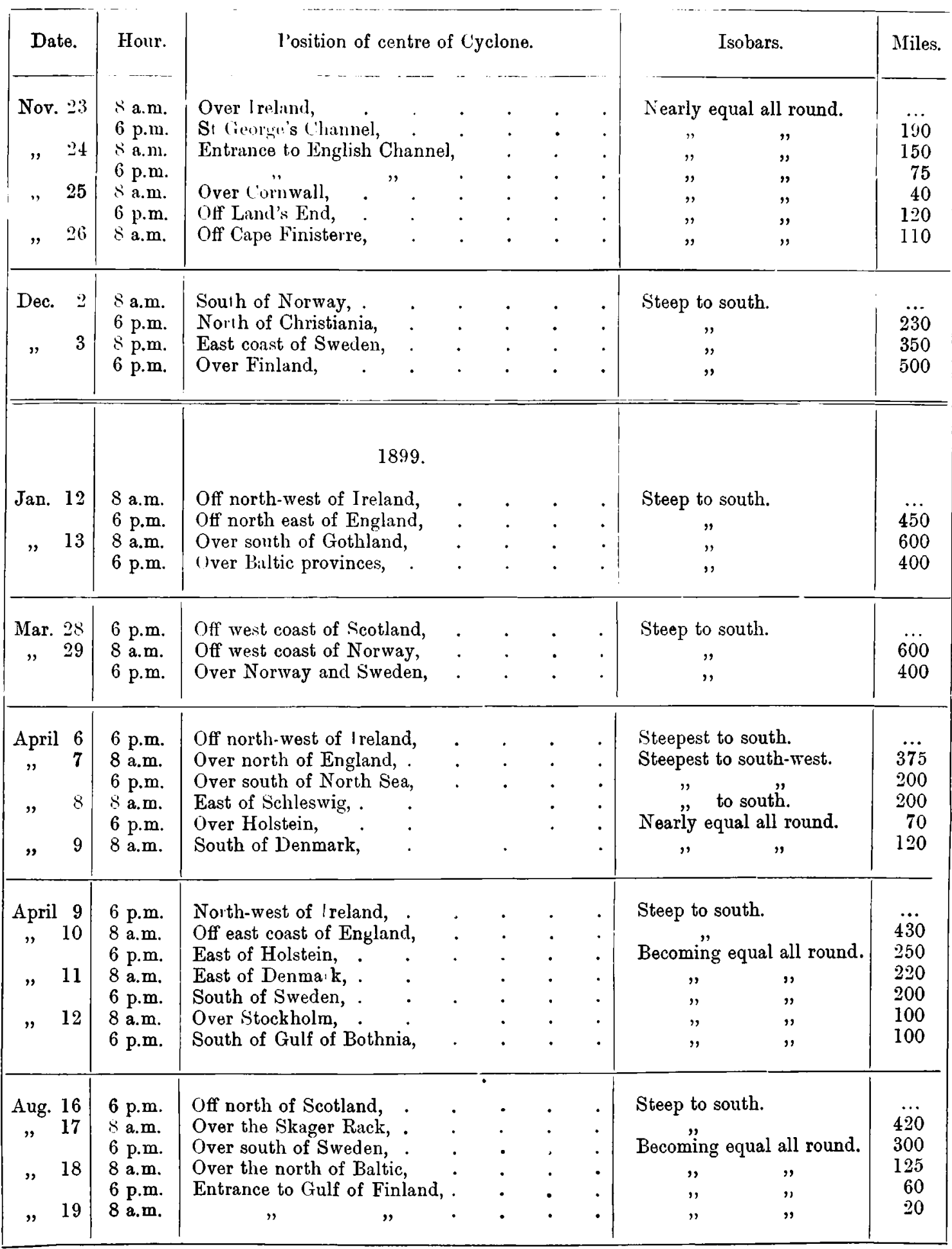


1899.

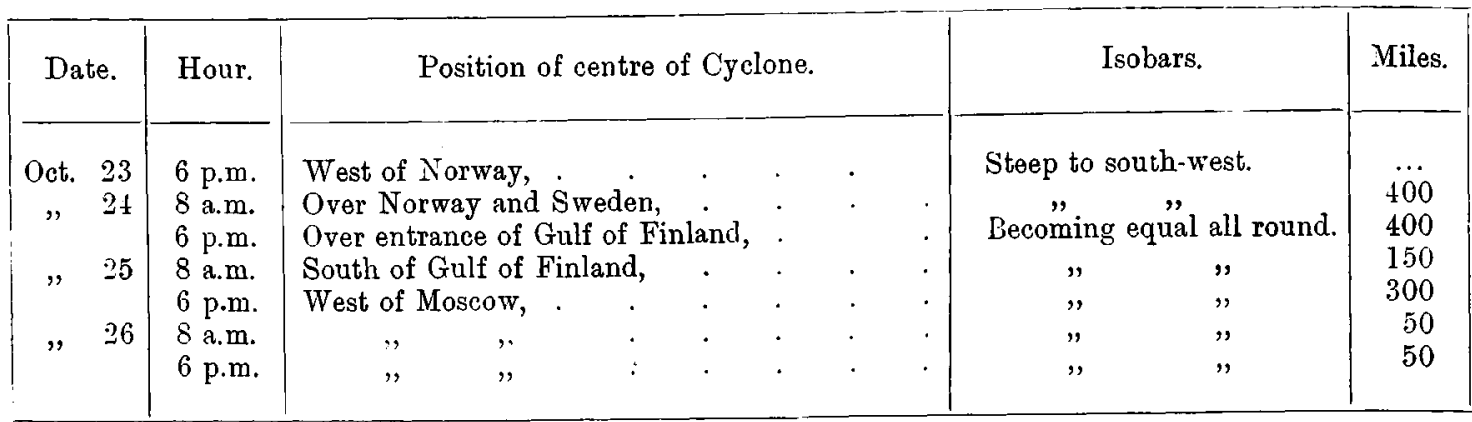

The history of all these cyclones bears out the conclusion that, when the barometric gradient is steep on one side, the cyclone travels quickly and nearly parallel with the isobars on the steep side, and that, as the gradients become equal all round the centre, the advance becomes slow.

Dr Buchan, in his Introductory Text-Book of Meteorology, points out that the isobars in cyclones are frequently eliiptical. Now that is just the form we would expect them to take when the barometric gradient was steep on one side only - that is, with strong winds blowing in one direction. The centrifugal force of these strong winds will tend to draw out the front of the cyclone, and make the length of the ellipse to point in the direction in which the centre of the storm is moving. When the winds are equally strong on all sides there is little tendency for the isobars to depart from the circular form, which an examination of the Synoptic Charts will show to be the case.

There is another effect of the inertia of the winds blowing along the steepest gradient which will be observed in these charts. Whilst the air from the anticyclone on the side next the cyclone is drawn into the cyclonic vortex, the air nearer the centre of the anticyclone passes on in the general anticyclonic circulation, and this quickiy-moving air in the anticyclonic area seems to force back the high pressure in front. The effect is to aid the advance of the cyclone by lowering the pressure in front of it. This effect will be seen in the chart for the 10th December 1898 (Plate I.). On the morning of the 9 th the shape of the isobars was similar to that shown in the chart for the 10th, but the furthest north point of the 29.9 isobar, instead of being near the eastern limit of our area, was a little to the north of Scotland, and it was driven eastwards to the position shown on the morning of the 10th. The curve of this isobar kept much the same form as it travelled eastwards.

We showed in the experimental part that the air currents at different elevations coming to the centre of the cyclone move in different paths, the air near the ground moving more radially than the air higher up, and it may be asked-Is there anything corresponding to this in the cyclones in the atmosphere? To get an answer to this question, the air currents in a number of cyclones have been examined. The position of the centre of the depression was taken from the Synoptic Charts, and the air 
currents were taken from the olservations marle by Me'ssis Bolam \& Redpath at Leith, along with those made by myself. The direction of the lower current is given by the wimls, whilst the direction of the ul' e' current is shown by the cloud movement. The result of this examination of the cycloms in the atmophere shows this feature in quite a distinct minnes. In all aises in which there was any difference in the recorded directions of the uple: and lower currents, the lower current flowed more directly towards the rentre of the depression than the upper. It was also noticed that the upper current was frrequently almost quite tangential, apparently not pointing at all to the centre of the depression. The observations used in investigating this point were almost all taken on the side of the depression on which the burometric gradient was steepest and winds strongest, and it would seem to indicate that the inflow towards the centre on this side-particularly of the upper air-is less than on the other sides. This result is indicated in the experimental part of the paper, where it is shown that the air on the side of the cyclone which receives the greatest tangential force curves inwards less than the air on the other sides.

The upper currents moving more tangentially than the lower ones has the effect, when a depression is passing any station, of making the upper currents appear to veer in advance of the lower. For instance, with a depression to the north of the point of observation, the wind will be south of west, whilst the cloud carry will be about west, and when the depression has passed eastwards the wind will change to west, whilst the carry will have some north in it. It may be as well to note here that it is possible that the upper part of the cyclonic column may travel in advance of the lower, owing to the friction on the earth's surface acting as a drag on the lower end. If this be the case it will in part explain the veering of the upper currents in advance of the lower.

We shall now turn our attention to anticyclones, and see what part they play in the vertical circulation of our atmosphere, as too little attention has, I think, been given to them. The sun's heat seems always to have been looked upon as the great source of the energy in our winds, to the exclusion of the effects of cold. It is as if, in studying a steam-engine, we had devoted our attention to the boiler and furnace, and neglected the condenser. The engine would no doubt work without the condenser, though not so efficiently, but it may be doubted whether heat alone rould work a cyclone without the cold-driven anticyclone. Its absence, at least, would be a greater loss to the vertical circulation than the loss of the condenser to the engine; unless, indeed, the engine was working at a very low pressure.

It is well known that the mean pressure over continental areas is high during winter and low during summer. That is just as the sun's rays during summer, by heating the air over continental areas, gives rise to cyclonic conditions; so the earth's radiation during winter gives rise to anticyclonic conditions, and this cooling of the air seems to be as true a cause of vertical circulation as the heating of it, though perhaps not to the same extent. If this be the case, then we ought to give more attention to 
the anticyclone than we have been giving, as a study of the area under its influence will probably assist in forecasting the movements of the cyclone and the weather conditions generally.

An examination of the weather charts for the winter months over our area shows that when the temperature is low over any part of it, particularly over the Spanish Peninsula and over the north-east of Europe, that as the temperature falls the pressure rises-that is, whenever the temperature falls below th ' mean, an anticyclone forms over the cold area, increasing in pressure as the cold strengthens. And, on the other hand, when the temperature begins to rise the pressure falls. It must be admitted that there are difficulties in studying this subject, as there is not at present sufficient information to enable us thoroughly to study the point. We would require a complete record of the clouds over the area, so as to enable us to judge how far the earth's radiation initiates the change. But $I$ think it may be accepted that, as a rule, any extreme cold over our area is generally accompanied by a small amount of cloud, and that the cold is due to radiation. An examination of the weather charts shows that, on some occasions, when the directive force of the cyclone was small, a rise of temperature in the anticyclonic area, by weakening the high pressure, caused the cyclone to move towards it. On the other hand, a decrease in temperature in the anticyclone may force the cyclone back. These charts also show that, if the temperature over both the Spanish Peninsula and the north-east of Europe falls very low at the same time, then the whole of Europe comes under anticyclonic conditions, the cyclones being driven from our area.

Let us now turn to the general question of cyclonic circulation, and see if what has been stated helps us to understand some of the other phenomena associated with cyclones and anticyclones. We have seen that the upper winds, circling from the anticyclones and to the cyclones, by moving more quickly, and by moving at an angle across the lower air, tends to prevent the latter rising, even though it be the lighter. The effect of this, as already pointed out, is to drive the hot moist air lying near the earth's surface to the circumference of the anticyclone, where it is picked up by the cyclone, and as the spirally moving cyclonic winds also tend to prevent the lower air rising, the hot, moist air is swept into the front of the low-pressure area, and the upper winds here cross the lower at a considerable angle, the hot moist air is compelled to keep near the ground, and, further, the air near the ground having a less tangential force, it is drawn into the centre of the depression, where it is drawn up, and as we saw in the experiments, forms the core of the cyclone. By this method of arranging the air in layers, the cyclone gets the best efficiency out of its material, the pressure falling from the circumference to the centre, whilst the temperature rises towards the centre of low pressure.

The effect of cyclonic circulation in keeping the hot moist air near the ground explains why the air in front of a cyclone is always hotter and moister than the air in the rear. The lower air sent forward by the anticyclone is swept off its 
circumference by the cyclone and drawn into the cyclone curving round the front of it, and in its passige it gets added to it all the hot moist air in front of the cyclonic area. After the cyclone is past it is evident that, as it has drawn up the surface air over the area of its trank, that what wiss previously the higher, chier, and purer air will have taken its place; hence the coolness, freshness, and purity of the air after the disturlance has pilssed. The eyclone has brought down to us the air we would have got if, before it passed, we had ascended a considerable distance from the earth's surface.

The above secms to give a fair explanation of the presence of the hot moist air in front of cyclones. Sitill, some may fecl inclined to say that is not the whole explanation, and that there is something else in the air orer that area which gives it a peculiar heavy feel-something, in fact, to which no hygroscope is sensitive. To exp]ain these peculiar sensations produced by this air, there is a point to which reference might be made. It is one which I do not think has ever been investigated or even referred to, and it is one which may hel, to explain the peculiar physiological effects experienced under these conditions. In front of a cyclone where the pressure in falling there will be a considerable amount of air rising from the soil and rocks underneath the surface. This air will come charged with moisture, and more or less changed in its composition by contact with the soil; it will also bring with it any impure gases there maly be in the soil. That such ail does rise there can be no doubt. If we observe the surface of the ground while the barometer is falling, after a slight fall of snow which has come before the frost, so that the temperature of the ground is not below the freezing point, we shall see little bare spots where the snow has melted. Is these little patches are scattered all over bare ground, gravel walks, etc., where the sulsuil is uniform, they can hardly be due to heat conducted upwards, but rather seem to be the result of hot air rising from the ground. The fact that the ground under these bare patches is more porous than elsewhere also points to the rising air is the cause of the melting. Though moist air rises from under the soil with a falling barometer, yet it is difficult to get any satisfactory way of measuring its amount. We can easily find out the air space in any sample of soil, and a few tests have been made in this direction. In place of taking the air capacity, the unoccupied space was measured by means of rater. A ylindrical vessel that held 17 litres of water was used. In making the tests this vessel was filled with the soil, firmly tramping in layer by layer: water wis then added till it showed on the top. In making this measurement it is necessiry to fill the vessel with water from below, otherwise it will not penetrate all through the soil. In the tests this was done by thrusting a small pipe clown through the centre of the soil, and pouring in the water at the top of the pipe. Well-packed garlen soil, tested in winter, when it was wet, gave the following result. In one case 3 litres of water were required to fill up the air spaces, and in another it required 3.4 litres, so that something between $\frac{1}{5}$ to $\frac{1}{6}$ of garden soil is air space. A clay soil would probably have less air space. When sand was treated in the same way, it required a little VOL. XL. PART I. (NO. 7). 
over 5 litres in one case and a little under 5 in another, which shows that about $\frac{3}{10}$ of sand is air space. The garden soil in the test vessel would probably be as. closely packed as the soil in the garden, but the sand would not be so firm as it was in its undisturbed condition, as I found it possible, by continued shaking while in the water, to make it more compact, and bring fully $\frac{1}{2}$ a litre of water to the surface.

These figures seem to indicate that about $\frac{1}{6}$ of cultivated soil is air space, and about $\frac{1}{4}$ is air space in consolidated sand. With these figures it would be easy to calculate the amount of air that each cubic foot would send to the surface with a given fall of the barometer. But this will not help us much, as it is difficult to get the air space in the lower undisturbed strata and find the mean depth from which the air rises. From the porosity of many soils it is evident that a considerable amount of air must ascend and descend with every change of pressure, and this air will have properties different from the air in the general circulation, and may give rise to certain physiological effects.

\section{PART II.}

(Read May 7, 1900.)

In the experiments described in the first part of this paper, the cyclonic morements were produced under very artificial conditions, the centre of low pressure being kept in a fixed position and the ascending column of air protected by solid walls. It seemed, however, that if the views set forth in Part I. were correct, that there was no reason why these experimental illustrations might not be extended, and made without much apparatus, and in free air, so as to allow us to study the motion of the centre of the depression as well as the spiral movements of the air towards it. On trial I found this could be easily done, and soon had the pleasure of seeing small cyclones forming and travelling across the experimental area, and the spirally inflowing air was seen moving towards the onward moving centre of low pressure.

The apparatus required for these experiments is very simple, and consists of a small platform, the surface of which can be heated for supplying the hot air required to make the cyclones. On this hot surface a wet piece of cloth or paper may be laid, or fumes may be formed, as in the previous experiments, to enable the eye to follow the movements of the air. Over this heated area, under certain conditions, cyclones are seen to form and travel across its surface. To enable the experimental surface to be heated, it was made in the form of a shallow tin box, closed on all sides and provided with two small pipes, one for the entrance of steam to heat it, and another for draining away the condensed water. This box is $75 \mathrm{~cm}$. square 
and $1 \mathrm{~cm}$. deep. This heated platform is placed on a table, and the boiler for producing steam to heat it at some distance away, so as to prevent the heat of the boiler producing disturbing currents near the experimental area.

When we watch this hot arei we will see the steam rising from its wet surface, and according to the draughts in the room, or absence of them, it will either rise up in an irregular manner, or le drifted across the hot area to the one side, but showing in neither case any tendency to form cyclonic movements. Suppose the air in the room to be perfectly still and the hot air ascending vertically. If we now blow gently over one side of the hot area, a cyclone at once forms which collects the hot air into its core and carries it to a considerable height. This cyclone soon dies away if we cease to supply the tangential energy.

Let us now see what the effect is if there is a draught across the hot area, and we alter the conditions by placing a screen across the current so as to shield one side of the hot area whilst it is allowed to blow over the other. A cyclone will now at once be formed at a short distance from the edge of the screen-a sort of eddy, in fact. It does not, however, remain in its place, but at once begins to travel across the hot area, in the same direction as the cross air current, rotating in the direction given by this tangential current, and rising to a considerable height above the platform.

It is not necessary that the screen protecting the one side of the platform should have a vertical edge, though such an edge is best suited for making eddies. The edge of the screen may be shaped like a magnified comb, as shown in fig. 7 . When so shaped the screen exerts very little effect on the current at the tips of the teeth, but more near their base, thus allowing a stronger current to blow over one side of the area than over the other. If this screen be put up across the current a cyclone will at once be seen forming near the points, and so soon as formed it begins to travel away across the hot area in the direction of the strongest tangential

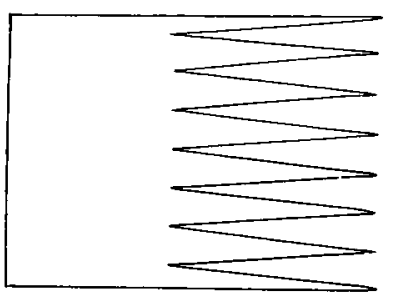

FIG. 7 . air current. Before this cyclone has gone far another forms near the screen, and it in turn passes, following the course of the first, and so on, cyclone following cyclone so long as there is a cross current and a heated area.

It may be as well to point out here that though there may be a slight tendency for eddies to form at the edge of the screen, in the above experiment, yet this tendency is very slight and has but little to do with the formation of the cyclones. If we repeat the experiment, without heating the experimental area, and using fumes to show the movements of the air, it will be found that only very slight eddying effects can be detected. Any that are formed with a screen having a vertical edge are small, irregular and broken, and frequently cannot be traced at all, and when the comblike obstruction is used no eddying effect is produced unless there is an ascending column of air. The question of the necessity of any eddying effect of the screen in 
starting the cyclonic morement in these experiments is disposed of by the experiment. previously described, in which it is shown that cyclones are formed in still air wher a horizontal current is made by blowing across one side of the ascending column, and without the intervention of a screen. The screen in these experiments is only used as a convenient method of protecting one side of the hot area from the horizontal current. If we wish to be more realistic we may crush up a piece of paper roughly into the form of a mountain ridge and put it in the place of the screen, with the result that cyclones go on forming as before.

When the air from the natural draught in the room flows over the hot area at the same velocity at all parts, the rising steam does not ascend far, but keeps close to the hot surface, and is irregular in its movements. But when a cyclone is formed, most of the steam is collected into a rapidly whirling vertical column, which ascends to a considerable height-to a metre or more above the hot surface, and often presenting the appearance of a well-defined column, as it rises through the clearer air.

In making these experimental cyclones, it was noticed there must be a definite relation between the amount of heating and the velocity of the cross current. If the heating be slight the cross current must be slow, otherwise the cyclonic movement will not be properly formed owing to the weakness of the ascent, before it is swept off the experimental area; whilst with hotter air a stronger current may be permitted, with the result that a more violent cyclonic movement is produced, which penetrates the upper air to a greater height.

The use of steam in these experiments is evidently open to many objections, other than the great amount of heat required to evaporate the water. Although it is water that plays a part in these experimental cyclones as well as in those in nature, yet in the two cases the part played by the water is reversed. In nature the water goes up as vapour, and when it condenses it liberates a great amount of heat, which prevents the temperature of the air falling so much as it would if no condensation took place, so aiding in the ascent of the column. But in the experimental cyclones this action is reversed, much of the water goes up in the cloudy or condensed form, and as in its ascent it gets mixed with more air, the water particles get evaporated and heat is absorbed. So that whilst the water taken up as vapour in the cyclones in nature increases the energy of the motion, it checks the movements in the artificial ones. Condensed water vapour or what we often call steam is thus not the best thing to use for showing these cyclonic movements, but it has the advantage that it is produced all over the experimental area, so that we can by means of it see the beginning and trace the movements of the cyclones. Fumes of hydrochloric acid and ammonia may be used, but in that case it is necessary to cover the experimental area with a sheet of glass, which is almost certain to crack with the heat. If the glass plate is not used the acid and ammonia may be put in watch glasses. Working with fumes we get a much greater violence of movement and greater ascent, owing to the air being more highly heated, but as the liquids evaporate very rapidly they require 
frequent renewal, and the rising fumes are patchy, thus allowing the core of the cyclone to be often invisible, and they do not often show a well-defined core of fumes.

These experiments are sometimes difficult to repeat with any degree of certainty, so much depends on the cross eurrents that happen to be in the room, which vary with changes in the outside temperature, winds, etc., and inside heating. For this reason it has been found advantageous to use some method of making a cross current that would be completely under control. An ordinary screw-shaped fan of $75 \mathrm{~cm}$. diameter does very well for the purpose. Owing to a fan of this construction not delivering its air in a uniform horizontal flow, but with an irregular somewhat spiral motion, it was placed at a distance of about $1 \frac{1}{2}$ metres from the hot area, and into the space between the fan and the hot area was fitted a borizontal surface, so as to ciuse the air to acquire a horizontal flow before arriving at the hot area. With this apparatus no difficulty has been experienced in repeating the experiments.

These miniature cyclones illustrate many of the points referred to in the first part of this paper. The slower rise of the air over the hot area when the circulation is uniform across it, that is, so to speak, anticyclonic, and the collecting together of the lower hot air, and the drawing of it up by the cyclone, and the power of the whirling column to penetrate the higher regions, is well shown. These small cyclones also illustrate the dependence of the spiral movements on the tangential current both for their velocity as well as direction of rotation. They also illustrate that the direction and the rate at which the cyclone as a whole travels is dependent on the direction and velocity of this tangential current.

If the cyclones be watched for a time whilst the draught in the room is not constant in direction or force, it will be seen that the stronger the tangential current the quicker the centre of depression moves along with it, and the more rapid is the rate of rotation, and if the draught slows down and reverses, then the direction of the movement of the centre of the cyclone also reverses, and it goes back on its track. Further, if there is no tangential force, as when there are no draughts across the area, then no cyclone is formed, and if formed during a temporary cross current it will die away and cease to rotate when the cross current ceases. Again, cross currents of equal force on opposite sides of hot areas keep the cyclone in one place and rapidly rotating.

It is not necessary to make special apparatus for making these experimental cyclones, as it will be found that a simple arrangement first tried is sufficient for the purpose. It consisted of a large sheet of tin plate; a thick sheet of iron would have been better. This was covered with two or three sheets of thick paper well soaked in water. This is placed over a stove or other means of heating it sufficiently to cause steam to rise freely from its surface. When steaming freely the wet surface is removed to a table, to be away from the draughts rising from the stove, and one side of the hot surface is protected from cross draughts by means of a screen. When this is done the cyclones will be seen travelling over the steaming surface. 
It seems probable that the small cyclones often seen in nature are produced under similar conditions to those above described. These small natural cyclones are often seen on dusty roads whilst the sun is shining, the whirling column having a core of dusty air, and the centre of motion travelling along the road, tossing up the dust and other light objects as it moves along. On one occasion 1 had the opportunity of seeing one of these small cyclones in a large park in early spring. The wind, which was very light, was cold and from the north, but the sun was very hot. The movements of the cyclone were easily seen as it tossed up the dry leaves in its path, whirling them up to a height of four or five feet and scattering them all round. The path of the cyclone was due south-that is, in the direction of the wind-the distance travelled being more than a hundred yards. As there were a number of large trees in the park, it was easy to see that the necessary conditions were present for starting and carrying on the cyclonic movement. It seems probable that these small cyclones are of more frequent occurrence than we are aware of, but owing to the absence of light bodies-such as dust, leaves, etc.-there is nothing to indicate their presence.

[Additional Note. Received February 19, and read March 4, 1901.]

If both the direction of revolution and the direction of movement of cylones as a whole are determined by the anticyclones, it may be asked-Why do cyclones over our area almost always move in an easterly or north-easterly direction, and so seldom in other directions? and why do these north-easterly moving cyclones generally travel so much quicker than those moving in other directions? The reason for so many cyclones moving in a north-easterly direction would appear to be due to there being generally a high-pressure area to the south of us, and accompanied with this, there is generally hot moist air coming from the Atlantic and blowing to the north of the highpressure area, and the cyclones form in this hot moist air and travel in the direction of the winds blowing out of the anticyclone lying to the south. That is, the frequency of these north-easterly moving cyclones is due to the conditions being more generally favourable for the formation of cyclones on the north-west side of the anticyclones, than on any of the other sides.

Turning now to the question of why these easterly moving cyclones should travel, as a rule, so much more quickly than those moving in other directions. It has been pointed out that the cyclone is formed in the low-pressure area between two anticyclones, and that it moves in the direction in which it receives the strongest tangential current. If a cyclone were situated between two equally strong anticyclones, it would receive equally strong winds on opposite sides, and it would not more, as it would receive equal amounts of air from both anticyclones, and as these equal amounts 
of air would have equal and opposite velocities, there would be no tendency for the centre of the cyclone to travel. But a little consideration will show us that the air to the north-western side of anticyclones, in our area, will generally have a greater velocity than the air on any of the other sicles. 'The high-pressure areas which regulate the morements of cyclones over our arcil are situated to the south-west of Spain, and over Siberia and northern Asia. Now, the air descending from high elevations over these areas has, in all probability, come from the upper general air circulation, and was previously moving from the equator towards the north pole. It will therefore have a greater casterly rate of motion than the surface of the earth where it clescends, on account of it coming from a lower latitude, and further, it will also have a northerly notion. The result of this is, that the air descending from the upper parts of the atmosphere, while it tends to move spirally outwards all round over the high-pressure area, yet owing to it having a northerly motion and a greater easterly motion than the surface of the earth where it descends, the air moving to the northwards and eastwards will have a much greater velocity relatively to the earth than the air which moves southwards and westwards. Further, over Europe the high-pressure area to the north is always much weaker than the one to the south, and is sometimes absent, so that the cyclone is always more influenced by the anticyclone to the south than the one to the north, and it thus receives its north-easterly motion from the strong winds on the northwest side of the southern auticyclone. These remarks only explain in detail how cyclones are affected by the general easterly drift of the atmosphere over our area.

An examination of the weather charts, however, shows that if a cyclone receives its strongest winds from any side of an anticyclone, it will move in the direction of these winds. If, for instance, it receives its strongest indraught from an easterly direction on the south side of the anticyclone, it will move to the west, or it may move northwards along the west side of the anticyclone, or southwards along its east side, but as the winds on these sides are generally feeble, the cyclonic movements are generally slow.

There is another point to which reference should be made here, as it has lately assumed considerable importance. For long it was held that cyclones were due to a a low-pressure area being formed by a rising column of hot moist air towards which the surrounding air flowed in spirally; while lately it has been contended that cyclones are only secondary effects due to the interaction of air currents, and are, in fact, eddies in the atmosphere formed by the general circulation of the earth's atmosphere. These two theories thus attribute the energy of cyclones to two different causes. According to the first theory the cyclone receives its energy from the hot and moist air; whilst according to the other it gets its energy from the general air circulation. The one theory we might call the convectional theory, the other the dynamical or driven theory. The question then comes to be, which of these two theories seems to be the most probable. To many it may seem difficult to imagine how the dynamical theory ever originated. The diameter of cyclones is so very great it is difficult to imagine any 
way in which the air currents in the atmosphere could produce eddies hundreds of miles in diameter.

Are there not, however, differences in these two forms of cyclonic movement by means of which we can distinguish the one from the other, and say whether the cyclones in our atmosphere are convectionally or dynamically driven? I think there are. One way in which we may distinguish between the two kinds is in the direction of the circulation. In a convectionally driven cyclone the circulation is spirally inwards, whereas in a dynamically driven one we would expect it to be spirally outwards. In a dynamically driven cyclone in our atmosphere we would be entitled to expect an inward current near the surface of the earth, owing to the velocity there being retarded by friction; the walls of the cyclone would therefore be weak at that part, and air would be drawn in at the surface of the ground, and it would also probably be drawn in at the top. Now, is there any evidence of a general outward circulation in cyclones with indraught at top and bottom?

For information on this point we cannot do better than turn to the valuable and very important results obtained by observations on clouds made by means of the nephoscope and theodolite, and published in the Report of the International Cloud Observations, prepared under the direction of WILLIS L. Moone, Chief of the Weather Bureau, by Frank H. Begelow, M.A., and published last year by the Weather Bureau, Washington, U.S.A. If we turn to the series of diagrams in the above Report showing the direction and velocity of the circulation in cyclones, we shall see that at only one cloud level does the circulation show any tendency to be outwards. The following is a summary of the results taken from the Report, pp. 435-6: "Beginning with the surface, it is seen (1) that all the vectors hare an inward component up to the cumulus, the inner increasing in strength ; (2) that they coutinue inward in the levels from the stratocumulus to the cirro-cumulus, except that there is a slight tendency to turn outwards on the exterior circle, the alto-stratus again showing average conformity with the others; (3) the tendency is still inward in the cirro-stratus and cirrus level in the interior, but apparently lawless over the outer parts." From the above it will be seen that at all levels except the strato-cumulus the circulation is inwards. This tendency to outward circulation at the one level is evidently due to the cyclone having at the strato-cumulus level its greatest rate of rotation, as shown by the diagrams, and this ligh velocity of rotation at this level would appear to be due to the air in the anticyclone also having its maximum velocity at the same level. Though the evidence here shows the direction of almost the whole of the circulation to be towards the centre, and thus points to the circulation being convectionally driven, yet as there may be some hesitation in accepting this evidence as final on the point, as all the circulation is not inwards to the top, I shall therefore now proceed to point out another and more definite way in which we can distinguish between dynamically and convectionally driven cyclones.

In a convectionally driven cyclone the velocity of movement of the air, both absolute and angular, increases from the outside towards the centre, whereas in a dynamically 
driven one the reverse is the case, the air in the outer parts moving quicker than the air in the inner. Judged by this test the evidence is entirely in favour of the theory that cyclones are convectionally driven. An examination of the diagrams in the Report of the Internationel Cloud (Hservenions: above referred to show in a marked way that in cyclones the velocity of the wind incruases towards the centre. These charts do not show any great increase of velocity towards the centre of the winds at the surface of the earth, but they show that as we ascend to greater elevations the increased velocity becomes more marked, and is very great at the strato-cumulus level, and up through the cirro-cumulus, alto-cumulus, alto-stratus, and into the cirro-cumulus level, a result we would not expect to find if cyclones were dynamically driven.

There is another point in connection with the source of energy in cyclones to which reference may be made here, and which seems to support the above conclusion. If cyclones are convectionally driven we would expect them to tend to form and move over areas where the air is relatively hot, or moist, or both. If we examine $\mathrm{Dr}$ BUCHAN's maps of the isobars and winds of the world, and his corresponding maps of the isotherms, and also Dunwoody's maps of the storm tracks of the world, all in Bartholomew's Physical Atlas, Vol. III., we will find this supposition receives considerable support. Confining our attention to the area over Europe and the eastern side of the Atlantic, it will be seen that in winter the general air circulation over this area is from the south-west, blowing out of the high-pressure area situated at this season to the south-west of Spain. It is this warm south-westerly wind that carries the high temperature at this season northwards to Iceland and Scandinavia, and gives rise to the upcurving over this area of the isothermal lines shown in the maps, and this hot moist air, driven northwards between the cold air on both sides, would seem to be the cause and source of energy of the cyclones which traverse this area during the winter months. The maps of the storm tracks show that the storms coming towards our area from the western Atlantic move generally along one track till they pass Newfoundland, where the track divides in two, and the storms travel over one of the two routes. A number of them go north-eastwards over Scandinavia, whilst the others move across England and eastwards over the Continent. Confining our attention to the more northerly route, it will be seen from the maps that during winter this track is at its furthest north position. In summer the conditions become somewhat changed, the general circulation does not blow so much from the south, the winds being at this season about west-south-westerly, and the isothermal lines no longer curve northwards to the extent they did in winter, and the track of the storms is now situated at its furthest south position, the relatively hot and moist air being now more to the south than it was in winter. From this it will be seen that the track of the cyclones over our area moves northwards as the isothermal lines curve northwards with the approach of winter, and again coming southwards when the isotherms tend to have an east and westerly direction, the storm track thus keeping over the relatively hot and moist area.

VOL. XL. PART I. (NO. 7). 
The movements of the southern storm track seem to be governed by the same law. In winter it attains its most southerly position, drawn southwards apparently by the hot moist air of the Mediterranean, over which area the storms move at this season, but when summer comes the continental area to the north becomes warmer than the Mediterranean, and the track of the storms moves northwards, and in summer is across coutinental Europe. It will be noticed that these two storm tracks move in opposite directions at the same season, the northern track moving south in summer and north in winter, while the southern one moves north in summer and south in winter.

It thus appears that storms tend to form and move over areas in which there is a supply of hot moist air, and to change their tracks so as always to follow the changes in the position of the best supply, and it is difticult to understand why this should be so, unless the hot moist air is the cause and source of energy in the cyclone.

Another consideration which leads us to suppose that convection currents play an important part in cyclones is the greater violence of the winds over cyclonic than anticyclonic areas, a result we would not expect to find unless some source of energy came into action in the cyclonic area. All these considerations point to the conclusion that however important the action of anticyclones may be in the formation of cyclones, yet cyclones are, to a very large extent, convectionally driven.

The general circulation over our area, as pointed out, is more from the south during winter than during the summer months. This change is partly brought about by the weakening during the latter season of the anticyclone to the south-west of Europe, but apparently much more to the disappearance of the anticyclone over Siberia and Northern Asia. In winter, Eastern Europe and Northern Asia are covered by a large and well-marked high-pressure area, and it would appear that it is this high-pressure area to the north-east which causes the anticyclonic circulation to turn more to the northwards in winter than in summer, so that the high winter air temperature and hot Gulf Stream water which carry the high winter temperature to Iceland and Scandinavia would appear to be greatly due to the high-pressure area over Siberia and Northern Asia. I fear I must apologise for adding one more theory to the many explaining the high winter temperature of the north-west of Europe. It seems strange that the cold over the northern parts of Europe and Asia should play any part in the abnormally high winter temperature of the sea and the lands surrounding the north-western parts of the Atlantic. 
Mr. Aitken on the Dynamics of Cyclones.

ARRANGEMENT OF ISOBARS IN QUICK MOVING CYCLONES.

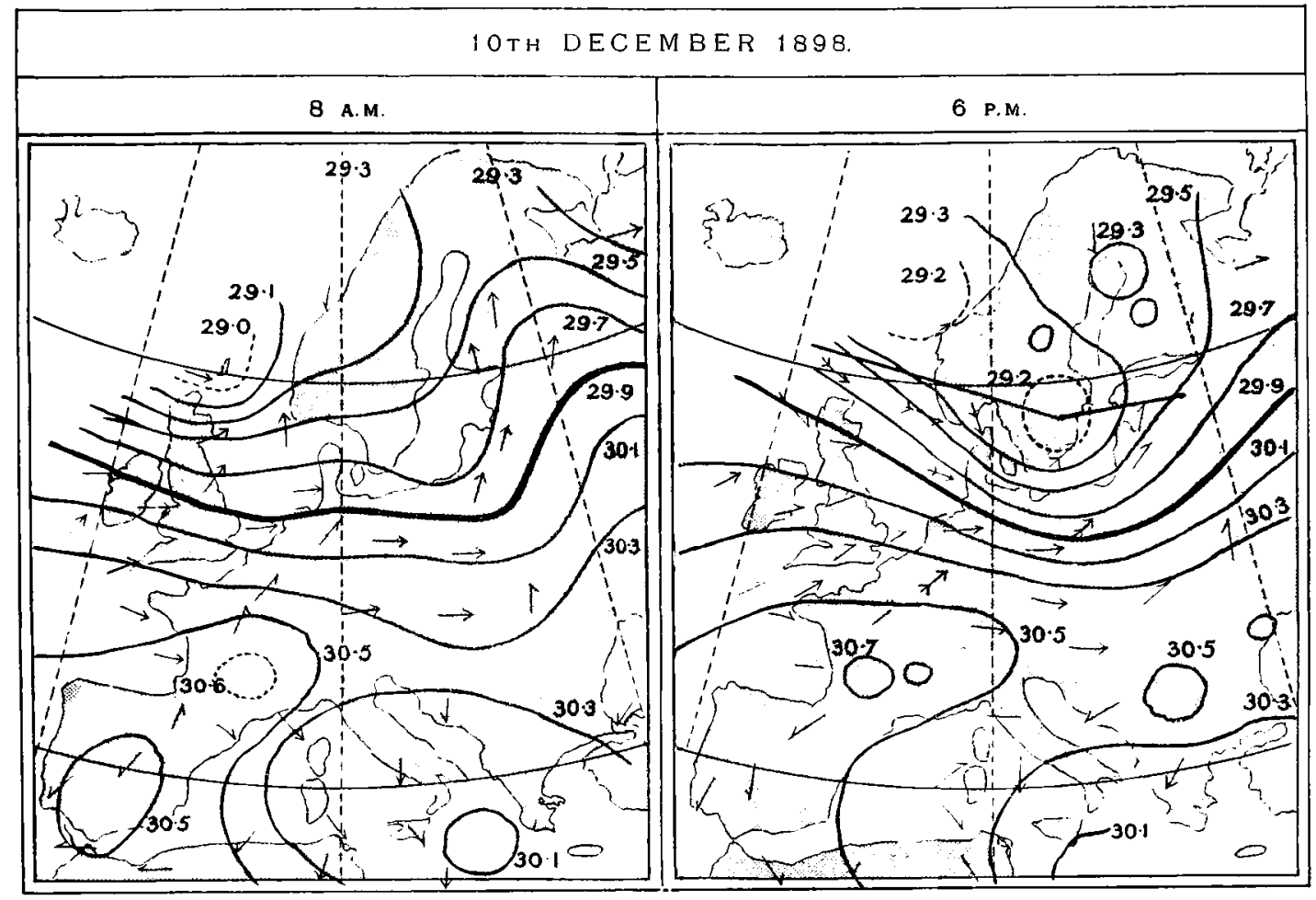

ARRANGEMENT OF ISOBARS IN SLOW MOVING CYCLONES.

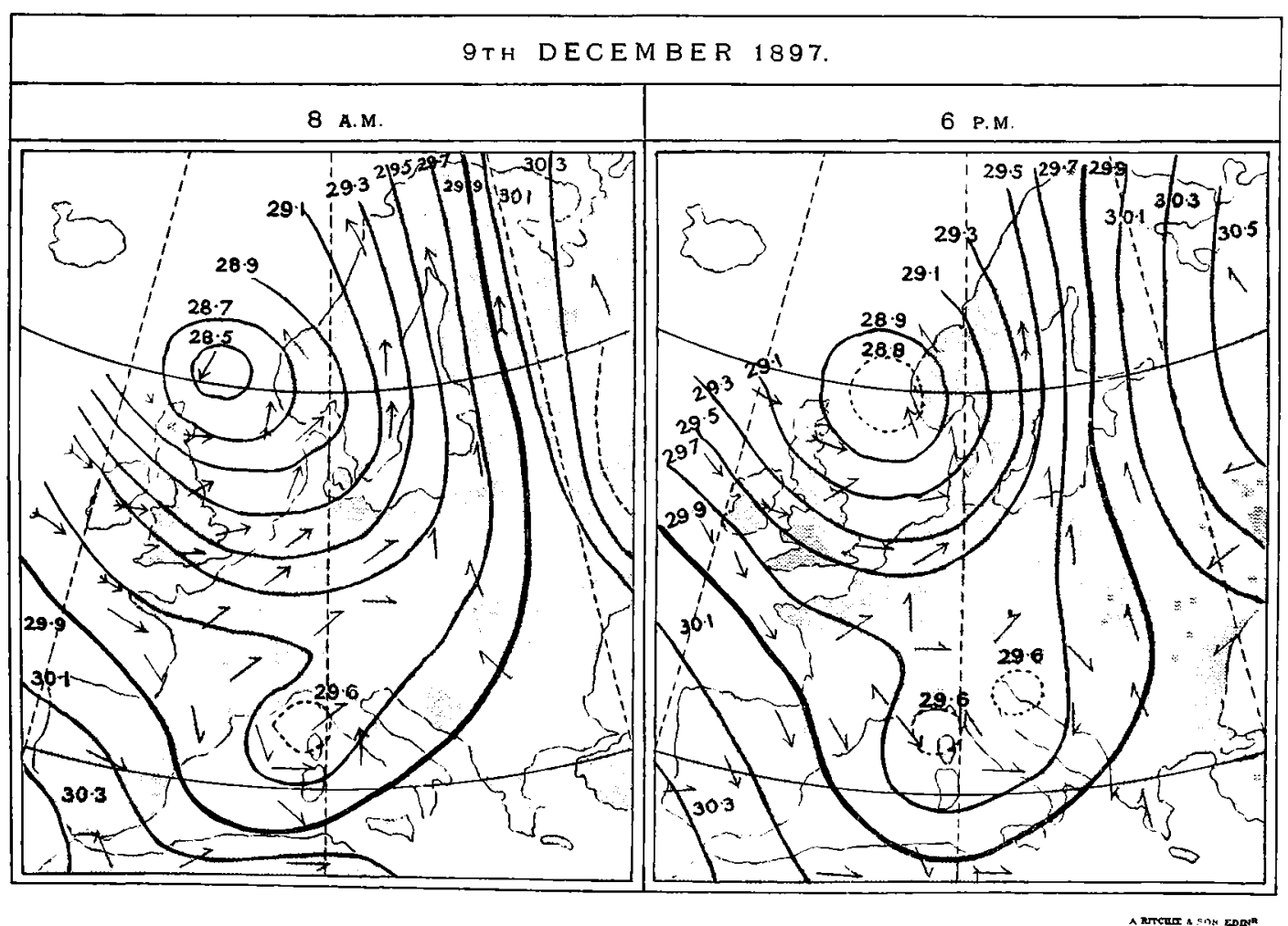

